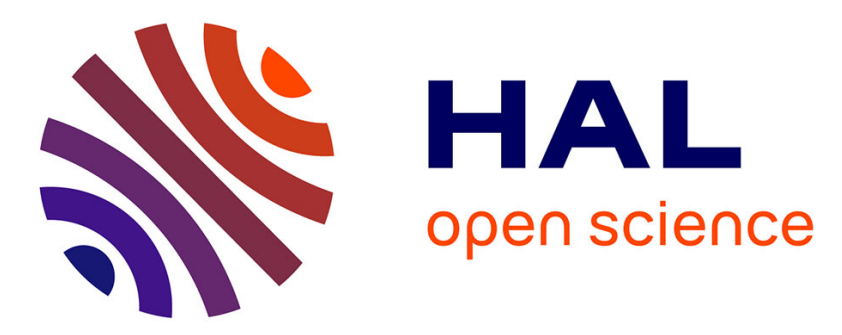

\title{
Delta-Fuzzy Similarity Entropy to Discriminate Healthy from Sick Fetus
}

\author{
Souad Oudjemia, Amira Zaylaa, Jamal Charara, Jean-Marc Girault
}

\section{To cite this version:}

Souad Oudjemia, Amira Zaylaa, Jamal Charara, Jean-Marc Girault. Delta-Fuzzy Similarity Entropy to Discriminate Healthy from Sick Fetus. 2nd International Conference on Advances in Biomedical Engineering, IEEE, Sep 2013, Lebanon. pp.1-4. inserm-00922140v2

HAL Id: inserm-00922140 https://www.hal.inserm.fr/inserm-00922140v2

Submitted on 13 Feb 2014

HAL is a multi-disciplinary open access archive for the deposit and dissemination of scientific research documents, whether they are published or not. The documents may come from teaching and research institutions in France or abroad, or from public or private research centers.
L'archive ouverte pluridisciplinaire HAL, est destinée au dépôt et à la diffusion de documents scientifiques de niveau recherche, publiés ou non, émanant des établissements d'enseignement et de recherche français ou étrangers, des laboratoires publics ou privés. 


\section{Delta-Fuzzy Similarity Entropy to Discriminate Healthy from Sick Fetus}

\author{
Souad Oudjemia \\ University of Tizi-Ouzou \\ Algeria \\ Email:souad.oudjemia@etu.univ-tours.fr
}

\author{
Amira Zaylaa, Jamal Charara \\ University Francois Rabelais of Tours \\ Signal and Image Laboratory, \\ Faculty of Sciences, \\ Lebanese University, Hadath, Lebanon \\ Email: amira.zaylaa@etu.univ-tours.fr \\ Email: jcharara@ul.edu.lb
}

\author{
Jean-Marc Girault \\ UMR "Imaging and Brain" \\ Faculty of Medicine \\ University Francois Rabelais of Tours \\ INSERM U930 \\ France \\ Email: jean-marc.girault@univ-tours.fr
}

\begin{abstract}
This paper deals with the discrimination between suffering and healthy fetuses, by means of a delta-fuzzy-similarity entropy. This new descriptor of complexity is based on the derivative of the fuzzy-similarity entropy. It was tested on fetal heart rate time-series and compared to the approximated and similarity entropies. The main outcome was the possibility to improve $10 \%$ the specificity and the sensitivity as compared to approximate entropy. This very good performance confirms that the new descriptor can be a valuable alternative as compared to other standard descriptors.
\end{abstract}

Keywords-delta-entropy, Entropy, Fuzzy, complexity, time series, suffering fetus.

\section{INTRODUCTION}

Analysis of fetal heart rate (FHR) is now a crucial step that is unavoidable for detecting the fetal distress. This analysis might help obstetricians to decide for immediate delivery by cesarean section but it might also be helpfull to better understand the fundamental reasons leading to foetal distress. This analysis was made difficult due to the wide range of possible pathologies and symptoms encountered during the gestational period.

A lot of work have shown the relevance of the complexity analysis of both human heart rate and fetal heart rate, and a huge number of indicators have been proposed [1], [2], [3], [4], [5], [6]. The most well-known descriptors were based on different kinds of entropy, as for instance, the approximate entropy or the sample entropy (SamEnt). These descriptors were devoted to characterize time series, and were used to measure the degree of regularity of the analysed time series. These measures were all based on the evaluation of the probability of finding similar $m$-patterns.

Recently a certain number of researchers has demonstrated the relevance of the similarity study of centered $m$-patterns [7]. This analysis consisted in calculating the probability of finding $m$-patterns located everywhere in the time series $x(n)$ and no more located on the same magnitude level of the time series. By doing so, true similarities were sought as exemplified in Fig. 1.

To improve the analysis of such similarity, this analysis can be advantageously completed by the introduction of a fuzzy

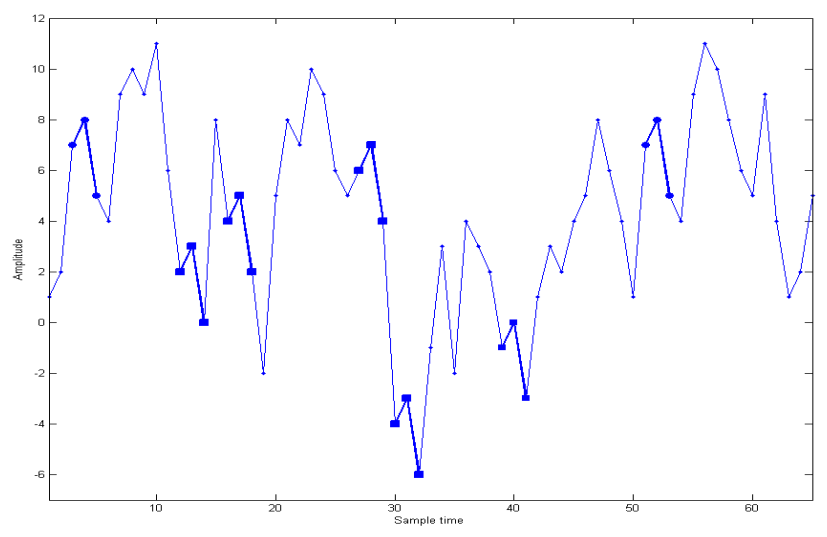

Fig. 1. By removing the baseline of each $m$-patterns, it was possible to count 7 similar $m$-patterns located everywhere in the time series whereas only $2 m$-patterns were accounted when the baseline was not removed.

membership function. This leads to the enhancement of the statistic stability as mentioned in [8], [9], [10].

Our study aimed to improve the differentiation between normal and suffering foetuses by deeply investigating the links between $m$-patterns. To do so, we investigated the fuzzy similarity entropy previously introduced by [9], [11] and we extended its definition to $\delta$-entropy.

We showed that it was possible through the delta-fuzzy similarity entropy method as compared to existing methods to better discriminate healthy fetuses from suffering foetuses.

To prove this, we compared our proposed method derived from the new $\delta$-entropy concept, to existing methods from our FHR dataset.

\section{METHOD}

The method that we have proposed aimed to evaluate the complexity of time series in a way to discriminate distressed fetuses to normal fetuses. The proposed method consisted in evaluating the entropy through the assessment of probability of finding the presence of $m$-patterns. 


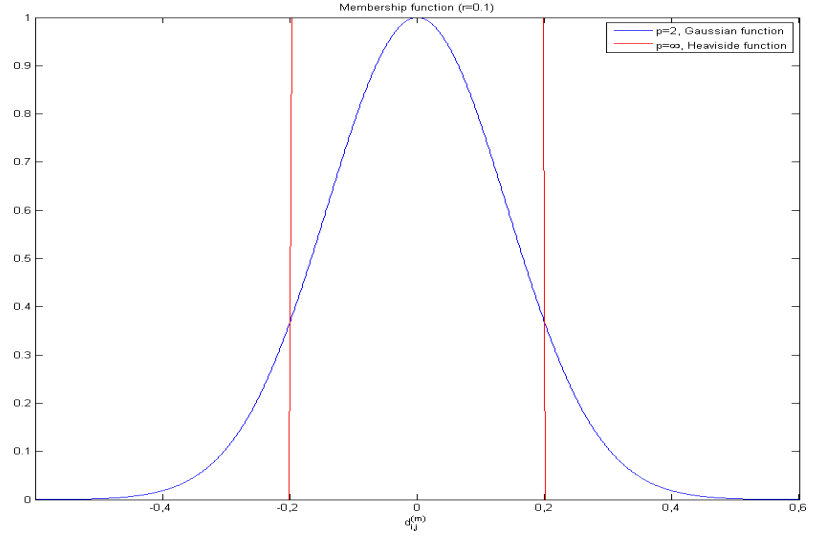

Fig. 2. Exponential membership function. The rectangular shape was obtained for $p=\infty$ and the gaussian shape was obtained for $p=2$.

From the time series vector $\mathbf{X}$ composed of 720 points, a vector sequence $\mathbf{X}_{i}^{(m)}$ was formed :

$$
\mathbf{X}_{i}^{(m)}=\{x(i), x(i+1), \ldots, x(i+m-1)\}-\overline{\mathbf{X}}_{i}^{(m)},
$$

with

$$
\overline{\mathbf{X}}_{i}^{(m)}=\frac{1}{m} \sum_{l=0}^{m-1} x(i+l) .
$$

These $m$-patterns $\mathbf{X}_{i}^{(m)}$ were formed directly from the original $m$ consecutive values extracted from the time series.

To catch the similarity of these two $m$-patterns $\mathbf{X}_{i}^{(m)}$ and $\mathbf{X}_{j}^{(m)}$, it was judicious to remove the baseline of each $m$ patterns. By doing so, all similar $m$-patterns located everywhere in the time series were accounted. In other words, instead of finding absolute $m$-patterns, all relative $m$-patterns were sought in the entire time series $x(n)$.

In this case, to account whether a vector $\mathbf{X}_{i}^{(m)}$ was similar to another vector $\mathbf{X}_{j}^{(m)}$ within a tolerance $r$, the correlation summation $C_{i}^{(m)}(r)$ was evaluated by:

$$
C_{i}^{(m)}(r)=\frac{1}{N-(m-1)} \sum_{j=1}^{N-m-1} \Theta\left(r, d_{i, j}^{(m)}\right) .
$$

$\Theta$ was a membership function, represented in Fig.2, defined as follows :

$$
\Theta\left(r, d_{i, j}^{(m)}\right)=e^{-\left(\frac{d_{i, j}^{(m)}}{r}\right)^{p}}
$$

and $d_{i, j}^{(m)}$ represented a distance between the two $m$-patterns $\mathbf{X}_{i}^{(m)}$ and $\mathbf{X}_{j}^{(m)}$ defined as follows:

$d_{i, j}^{(m)}=d\left(\mathbf{X}_{i}^{(m)}, \mathbf{X}_{j}^{(m)}\right)=\max _{k \in(0, m-1)}|x(i+k)-x(j+k)|$.

When the family of exponential function $\exp \left(-\left(d_{i, j}^{(m)} / r\right)^{p}\right)$ was selected as a membership function, the parameter $p$ needed to be fixed. When $p$ tends to infinity the membership function tended to the Heaviside function while $p=2$ the membership function was gaussian.

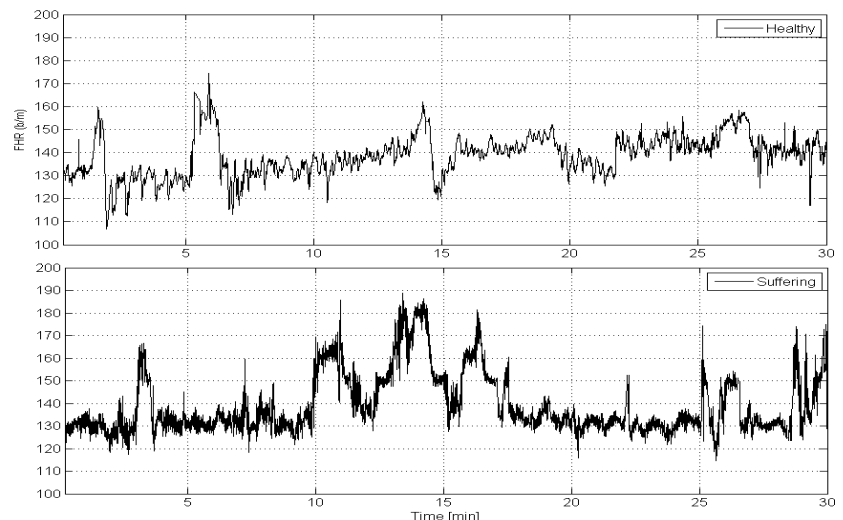

Fig. 3. Traces of two fetal heart rate (FHR). (Top) FHR of a healthy fetus. (Bottom) FHR of distressed fetus. FHR of distressed fetus varied more rapidly than the healthy fetus.

We defined a new function $\delta(m, k)$ based on the difference between $\Phi^{(r)}(m)$ and $\Phi^{(r)}(m+k)$ as follows:

$$
\delta(m, k, r)=\frac{\left(\Phi^{(r)}(m)-\Phi^{(r)}(m+k)\right)}{k}
$$

where

$$
\Phi^{(r)}(m)=\frac{1}{N-(m-1)} \sum_{i=1}^{N-(m-1)} \log C_{i}^{(m)}(r)
$$

Note that the approximate entropy is a special case of equation (5) when $k=1$.

To sum up (see Table I), six entropy indicators were considered here: three existing descriptors such as approximate entropy (AppEnt), similarity entropy (SimEnt) and fuzzy similarity entropy (FuzzySimEnt) and three new $\delta$-descriptors such as $\delta$-approximate entropy ( $\delta$-AppEnt), $\delta$-similarity entropy ( $\delta$ SimEnt) and $\delta$-fuzzy similarity entropy ( $\delta$-FuzzySimEnt).

\begin{tabular}{cc||c|c} 
& & $k=1$ & $k>1$ \\
\hline baseline removed & $p=\infty$ & SimEnt & $\delta$-SimEnt \\
\hline baseline removed & $p=2$ & FuzzySimEnt & $\delta$-FuzzySimEnt \\
\hline baseline & $p=\infty$ & AppEnt & $\delta$-AppEnt
\end{tabular}

TABLE I. SUM-UP OF THE DIFFERENT ENTROPY TECHNIQUE USED.

\section{APPLICATION TO FETAL HEART RATE TIME SERIES}

\section{A. Protocol}

Our database included 130 FHR traces which were collected from our home-made ultrasound Actifoetus Unit in $\mathbf{6 5}$ pregnant women. The women were in a gestational age ranging from 25th and 39th week. Recordings were obtained at CHRU "Bretonneau, Tours, France. The consent of each patient was obtained and the study was approved by the ethic committee of the Clinical Investigation Center for Innovative Technology of Tours (CIC-IT 806 CHRU of Tours). 

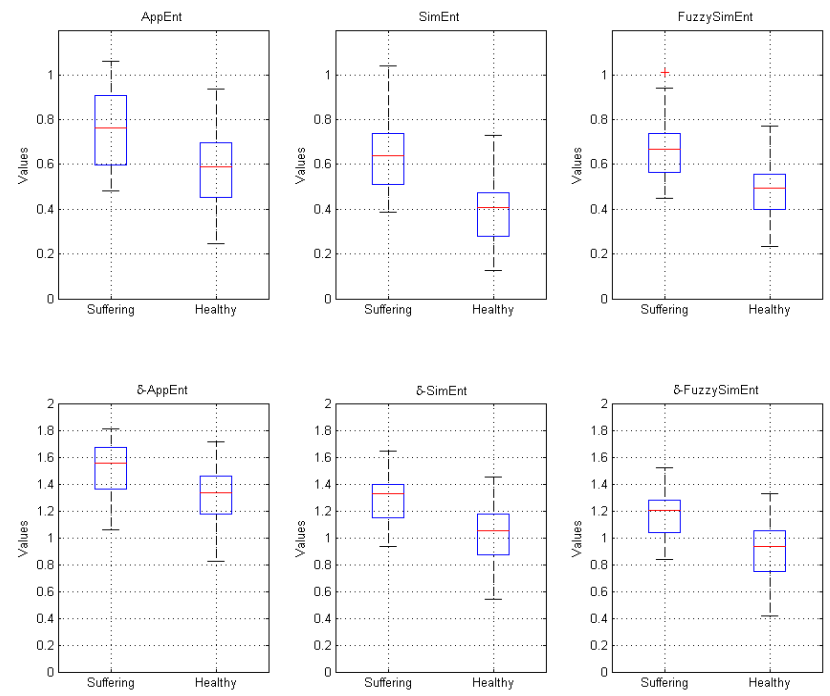

Fig. 4. Boxplot of different entropies for suffering and healthy fetus. Standard indicators were obtained for $m=2, k=1, r=0.2$ while $\delta$-indicators were obtained for $m=2, k=2, r=0.2$. (Top-left) approximate entropy (AppEnt). (Top-middle) similarity entropy (SimEnt). (Top-right) Fuzzy similarity entropy (FuzzySimEnt). (Bottom-left) $\delta$-approximate entropy $(\delta$ AppEnt). (Bottom-middle) $\delta$-similarity entropy ( $\delta$-SimEnt). (Bottom-right) $\delta$ Fuzzy similarity entropy $(\delta$-FuzzySimEnt).

The data for each subject consisted of $30 \mathrm{~min}$ FHR recordings composed of 7200 points. Two recordings of 30 min were obtained for each women. FHR was evualated as proposed by [12].

For each FHR trace, entropies were short-term evaluated through a sliding window of 720 points with an overlap of 97\%. Two FHR traces were depicted in Fig. 3.

Fig. 3 (Top) shows the FHR of a normal fetus while Fig. 3 (bottom) shows the FHR of a distressed fetus. Note the strong FHR variability of the suffering fetus as compared to the healthy fetus.

In this study, we considered two groups of fetuses: a control group of $\mathbf{4 7}$ normal fetuses (absence of pathologies at birth, no antepartum problems and spontaneous delivery) and a group of 18 suffering fetuses, i.e. intrauterine growth-restricted fetuses.

\section{B. Results and discussion}

As previously mentioned, six indicators were tested from our own dataset. Among all possible values of $m$, the best performances were obtained for $m=2$. The tolerance value was set at $r=0.2$ as its usual value. For the first three descriptors the parameters were setting to $m=2, k=1$, $r=0.2$. Among all possible values of $k$, the best performances were obtained for $k=2$. For this condition and for the last three $\delta$-descriptors the parameters were setting to $m=2$, $k=2, r=0.2$.

A box-plot of each descriptors were reported in Fig.4 for both suffering and healthy fetuses. Fig.4 shows entropy values higher for suffering fetuses than for healthy fetuses whatever the entropy descriptor used. This outcome means

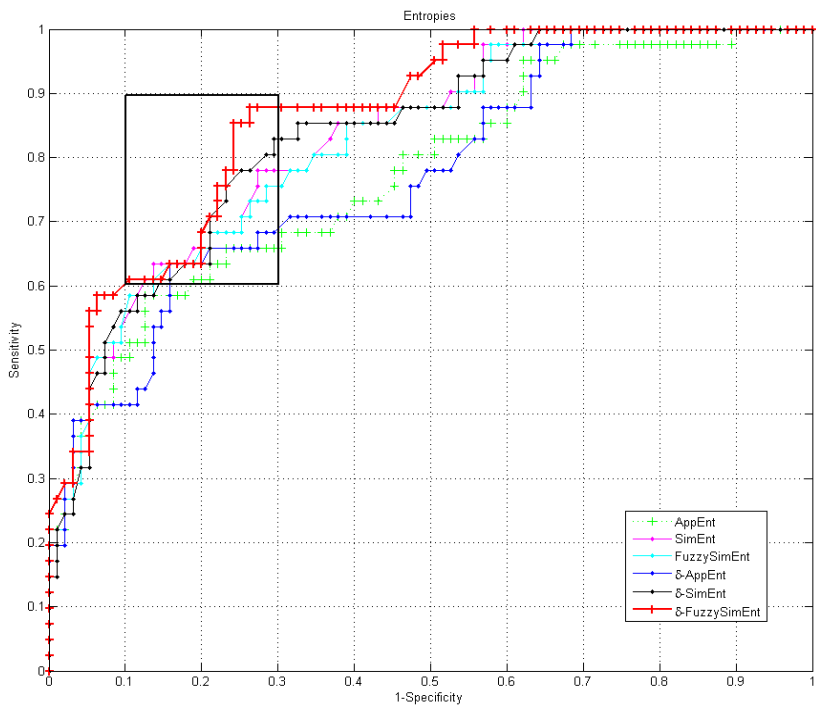

Fig. 5. Receiver Operating Curve for each methods: Probability of detecting pathological fetuses versus probability od detecting normal fetuses. Standard indicators were obtained for $m=2, k=1, r=0.2$ while $\delta$-indicators were obtained for $m=2, k=2, r=0.2$. The best curve corresponding to the thick curve was obtained for $\delta$-FuzzySimEnt and the worst curve corresponding to the red curve was obtained for AppEnt.

that the suffering fetuses had a higher complexity than healthy fetuses. This result seemed to be corroborated by the more FHR fluctuating nature of the Suffering fetuses than healthy fetuses (see Fig.3).

Fig.5 shows the sensitivity versus 1 -specificity for the 6 different entropy methods. Note that an ideal method will have a sensitivity of $100 \%$ and a specificity of $100 \%$ which corresponds to a point located in the left-top corner of Fig.5. Interesting performances are located in the black rectangle in Fig.5, as for instance, $\delta$-FuzzySimEnt gives a sensitivity of $86 \%$ and a specificity of $76 \%$ while AppEnt gives a sensitivity of $70 \%$ and a specificity of $61 \%$. The best method is the $\delta$-FuzzySimEnt and the worst is AppEnt. Note all methods, whose baseline have been removed, give good results. Furthermore, in all cases the $\delta$ versions ( $\delta$-AppEnt, $\delta$-SimEnt, $\delta$-FuzzySimEnt) overpass all methods in their standard form.

This novel finding seems to be an alternative solution when the discrimination seems to be so hard. In this study, performances could have been improved $10 \%$ in terms of both sensitivity and specificity.

Methods based on $\delta$-Entropy seems to have the potential to overpass all existing entropy methods, however this behavior has to be confirmed for another kind of data.

\section{ACKNOWLEDGMENT}

The authors would like to thank Prof. F. Perrotin for his support in the data collection process at the Obstetrical Department, CHRU Bretonneau, Tours, France. 


\section{REFERENCES}

[1] Pincus, S.M. 'Approximate entropy as a measure of system complexity', Proceedings of the National Academy od Sciences of USA, Vol. 88, pp. 2297-2301, 1991.

[2] Richardman, J.S. and Moorman, J.R. 'Physiological time series analysis using approximate and sample entropy', American Journal of Physiology: Heart and Circulatory cPhysiology, Vol. 278, pp.H2039-2049, 2000.

[3] Bandt, C. and Pompe, B. 'Permutation Entropy: a natural complexity measure for time series', Phys. Rev. Lett., Vol. 88, pp. 174102, 2002.

[4] Costa, M. and Goldberger, A.L. and Peng, C.-K. 'Multiscale entropy analysis of complex physiologic time series', Phys. Rev. Lett., Vol. 89, pp. $068102,2002$.

[5] Ferrario, M. and Signorini, M.G. and Magenes, G. and Cerutti, S. 'Comparison of Entropy-Based Regularity Estimators: Application to the foetal Heart Rate Signal for the Identification of foetal Distress', IEEE Trans. BME, Vol. 53, pp 119-125, 2006.

[6] Ferrario, M. and Signorini, M.G. and Magenes, G. 'Complexity analysis of the foetal heart rate variability: early identification of severe intrauterine growth-restricted foetuses', Med. Eng. Comput., Vol. 47, pp 911-919, DOI 10.1007/s11517-009-0502-8, 2009.

[7] Ning X. and Xu Y. and Wang J.and Ma X. 'Approximate entroopy analysis of short-term HFECG based on wave mode', Physica A, Vol. 346, pp. 475-483, 2005.

[8] Chen W. and Wang Z. and Xie H. and Yu W. 'Characterization of surface EMG signal based on fuzzy entropy', IEEE Trans. Neural Systems and Rehabilitation Engeineering, Vol. 15, pp. 266-272, 2007.

[9] Xie, H.-B. and He, W.-X. and Liu, H. 'Measuring time series regularity using nonlinear similarity-based sample entropy', Physics Letters A, Vol. 372, pp. 7140-7146, 2008.

[10] Liu C. and Li, K., Zhao, L. and Liu, F., Zheng D. and Liu C. and Liu S. 'Analysis of heart rate variability using fuzzy measure entropy', Computers in biology and Medicine, Vol. 43, pp. 100-108, 2013.

[11] Voicu, I. and Girault, J.-M. 'Multi-scale Similarity Entropy as a New Descriptor to Differentiate Healty to Suffering foetus', International Conference on Complex Systems, 5-6 Nov. Agadir-Morocco, 10.1109/ICoCS.2012.6458605, 2012.

[12] Voicu, I. and Girault, J.-M. and Menigot, S. 'Improved estimation of the foetal heart rate using directional Doppler signal and YIN', IRBM, Vol. 33, pp. 262-270, 2012. 